\title{
Percepción de la lepra y las discapacidades antes del diagnóstico en Recife, Brasil ${ }^{1}$
}

\author{
Katia V. de O. Feliciano, ${ }^{2}$ Maria Helena Kovacs, ${ }^{2}$ \\ Elías Sevilla ${ }^{3}$ y Alberto Alzate ${ }^{4}$
}

RESUMEN Este artículo presenta un estudio de casos y controles realizado en Recife, Brasil, entre noviembre de 1993 y julio de 1994. En él se investigó cómo influyen la percepción y las apreciaciones de los propios pacientes de lepra en el proceso de manejar la enfermedad y en la utilización de los servicios de salud. La muestra estuvo constituida por 183 pacientes de 20 a 70 años de edad, residentes en Recife, que acudieron en busca de un diagnóstico a los servicios de dermatología sanitaria de dos centros de referencia de las regiones politicoadministrativas tercera, cuarta y sexta. Se clasificaron como casos los 64 pacientes que tenían discapacidades o lesiones precursoras de discapacidad; los 119 restantes se consideraron controles. Todos fueron diagnosticados durante el período de la investigación. En el análisis se ajustó según sexo, edad, escolaridad y antecedentes de la enfermedad de Hansen de los pacientes.

El estudio reveló la coexistencia de dos tipos de "invisibilidad" de la enfermedad en una zona endémica en expansión: 1) para los pacientes de ambos grupos, la baja frecuencia de modelos explicativos, espontáneos, relacionados con la dolencia, aun en presencia de antecedentes de la enfermedad, y 2) para los profesionales sanitarios, las limitaciones de la detección. Puesto que afectan a las decisiones relacionadas con el manejo individual y colectivo de la enfermedad, esas deficiencias constituyen por sí mismas un factor de riesgo y representan un obstáculo para la eliminación de la lepra como problema de salud pública.

En las zonas urbanas, las características de la organización social del espacio y las condiciones materiales existentes afectan la dinámica poblacional de enfermedades como la lepra (1). En Recife, Brasil, desde 1988 se ha

1 Investigación financiada por el Programa Especial de Investigaciones y Enseñanzas sobre Enfermedades Tropicales PNUD/Banco Mundial/OMS (Proyecto ID-920523).

2 Universidad de Pernambuco, Facultad de Ciencias Médicas, Pernambuco, Brasil. Dirección postal: Rua Arnóbio Marques, 310, Santo Amaro, Recife, Pernambuco, CEP 50100-130. Fax: 55-081-4233371.

3 Universidad del Valle, Facultad de Ciencias Sociales y Económicas, Cali, Colombia.

4 Universidad del Valle, Facultad de Salud, Cali, Colombia. registrado un aumento sistemático de las tasas de detección de la enfermedad en ambos sexos independientemente de la edad, sobre todo las de las formas clínicas tuberculoide y dimorfa $(2,3)$. Esta situación incrementa el riesgo de desarrollo precoz de discapacidad, ya que no puede garantizarse un diagnóstico temprano o un tratamiento adecuado.

La experiencia de cada individuo está sometida a la influencia de las relaciones sociales en las que se desarrolla su vida (4). Por lo tanto, las decisiones que toman los enfermos en cuanto a dónde y cuándo buscar ayuda o acudir a algún centro de salud en especial dependen de los conocimientos que tienen sobre su enfermedad y de la experiencia acumulada en el seno de la familia y en las redes de apoyo sociales e institucionales. Estas desempeñan un papel importante, pues condicionan los valores de los enfermos y sus necesidades de salud (4-7).

La imagen social de la lepra sigue asociándose estrechamente con el desarrollo de deformidades físicas, aunque la matriz cultural de su estigmatización en el pasado incluía también la incurabilidad y la contagiosidad como creencias que contribuían al mantenimiento de fuertes prejuicios contra los 
enfermos. Se interconectan así diversas percepciones que condicionan la representación social de la enfermedad como sufrimiento y peligro (8). Estas son las limitaciones físicas, las dificultades y ansiedades que la dolencia provoca en la vida familiar y social, la conciencia de que la dolencia puede transmitirse, la enfermedad como prueba del castigo divino, y demás.

En los últimos años se ha implantado una estrategia para desmitificar la enfermedad que se basa en sustituir la denominación lepra por la de enfermedad de Hansen (hanseníase en el Brasil) y hacer hincapié en que actualmente hay medicamentos eficaces para su tratamiento (9). No obstante, sigue existiendo una distancia entre la lógica práctica que apoya las acciones de los pacientes y de sus redes sociales en el manejo de la enfermedad y de su riesgo potencial de provocar discapacidad $(10,11)$ y la lógica técnica que trata la enfermedad como una simple entidad biológica contra la cual es posible usar una tecnología poderosa (12).

En la lepra, el amplio espectro de respuestas inmunitarias que desarrollan las personas infectadas y que se manifiesta en los signos y síntomas peculiares de las distintas formas clínicas (13-17), la percepción de vulnerabilidad personal, la imagen social y los efectos que estas tienen en las actitudes de otras personas hacia los enfermos $(18,19)$ condicionan las distintas intensidades con que la enfermedad afecta a las relaciones personales y profesionales. Esos aspectos llevan a asumir distintas conductas adaptativas. Por lo tanto, las características de las manifestaciones corporales, las explicaciones desarrolladas para justificarlas y el temor de sus consecuencias pueden reducir la significación del problema en función de otros riesgos y necesidades competitivas, que resultan más urgentes desde una perspectiva existencial global (20).

Para garantizar la efectividad de las acciones puestas en marcha, los modelos especializados del funcionamiento de los procesos de salud se fundamentan en el conocimiento técnico del pro- blema y en el desarrollo de actividades específicas en la red jerarquizada de atención de salud. Sin embargo, no tienen en cuenta que en la vida cotidiana las necesidades se interpretan en distintos contextos socioculturales y las acciones concretas se basan en la lógica práctica que surge de la interacción entre las experiencias previas, los significados, los sentimientos, las motivaciones y las conveniencias prácticas de los individuos y de los grupos sociales $(4,7,12,21,22)$.

En algunos estudios que comparan el razonamiento popular con el técnico en la adopción de medidas para resolver problemas específicos de salud, se ha visto que entre los individuos de nivel socioeconómico alto el manejo de las cuestiones de salud presenta una mayor aproximación a la lógica técnica, si bien dentro de cada grupo las personas más convencidas de los beneficios de buscar atención tienen mayor probabilidad de hacerlo (19, 23). También se ha visto en esas investigaciones que las personas en peores condiciones socioeconómicas dan prioridad a los resultados más inmediatos en detrimento de los objetivos a mediano plazo, lo que sugiere que esa diferencia en la orientación temporal de las distintas clases sociales puede tener consecuencias para el planeamiento de las acciones de salud.

Así, el acceso de la población de enfermos de lepra a los servicios sanitarios podría hacerse viable por la forma en que esos servicios se organizan para producir y distribuir las acciones de salud, pero su verdadera utilización representa un grado de ajuste entre las experiencias, percepciones y valores de esta población y el modo en que las acciones de salud se producen $(7,24)$. Kroeger, en su revisión de los principales modelos empleados para el análisis de las conductas relacionadas con la búsqueda de atención de salud (25), observó la utilización de dos tipos de modelos, uno que considera los caminos de decisión y otro, los determinantes del uso de los servicios de salud. En este trabajo se parte del supuesto de que el estudio cuidadoso de las relaciones entre las manifestaciones somáticas presentadas por los pacientes de lepra, sus explicaciones sobre el nuevo fenómeno, la experiencia previa respecto a la enfermedad, el apoyo de las redes sociales y el manejo institucional de la dolencia nos permitiría identificar diferencias de trayectoria que llevan a los pacientes, con o sin discapacidades o lesiones precursoras de discapacidad, hacia el diagnóstico.

En nuestro enfoque del problema en Recife, utilizamos el modelo de interpretación de las decisiones humanas propuesto por Allen (26) para verificar la influencia de la percepción desarrollada por los pacientes y su evaluación del problema en el proceso de manejar la enfermedad y acudir a los servicios de salud. En ese modelo se considera un juego de interacciones entre tres fases principales: 1) una fase de creación de modelos para interpretar la realidad: al percibir y evaluar la aparición de modificaciones en el propio organismo, el individuo intenta identificar y explicar esas modificaciones basándose en la experiencia previamente acumulada; 2) una fase de anticipación de los posibles futuros: se prevén las consecuencias físicas y sociales de las lesiones, imaginando los pros y los contras de las distintas opciones para resolver el problema; y 3) una fase de evaluación de las perspectivas futuras según los valores personales y las decisiones para actuar y cuya combinación puede llevar o no a una conducta concreta cuando alcanza una elevada intensidad. Este proceso es iterativo y no sigue una secuencia necesariamente lineal.

Basándonos en los recursos técnicos de la antropología y la epidemiología, seguimos la línea de trabajo de Laplantine (27) para examinar la enfermedad en primera persona, ya que fue lo percibido $y$ vivido por el enfermo lo que nos señaló el juego alternado que existe entre lo manifiesto y lo latente. Este enfoque no pretende desarrollar una culpación de la víctima (28), en la que la individualización de la culpa resulta en una explicación de una práctica de la colectividad. Los valores que rigen la interpretación de las necesidades no provienen de una experiencia monológica (29); su historia guarda relación íntima 
con los discursos y prácticas de una sociedad concreta $(22,30)$.

Las principales limitaciones de este estudio son la exclusión del curso de acción de los enfermos que no tuvieron acceso a los servicios de salud, su carácter retrospectivo y su acotamiento regional. A pesar de las posibilidades de producir resultados que son culturalmente específicos, sus posibilidades de aplicación están abiertas a otros grupos y lugares que necesitan considerar el mismo problema. El carácter retrospectivo de la investigación podría implicar un sesgo de memoria y la aparición de algunas imprecisiones y vacíos en la información; sin embargo, era fundamental interrogar sobre hechos que habían ocurrido en el pasado. Para Bachelard (31) "el único medio de analizar una acción es repetirla, y es entonces preciso recomenzarla por medio de su descomposición, es decir, enumerando y ordenando las decisiones que la constituyen".

A pesar de las limitaciones de nuestro enfoque, esperamos que los resultados puedan contribuir a apoyar concretamente el desarrollo de modelos de intervención más eficientes, efectivos y justos, en la medida en que permitan identificar algunas particularidades del proceso diagnóstico de los pacientes que tienen la enfermedad de Hansen y en que faciliten una mayor compatibilidad entre estos y las acciones de los servicios de salud.

\section{MATERIALES Y MÉTODOS}

Este estudio de casos y controles de carácter exploratorio se llevó a cabo en Recife desde el 3 de noviembre de 1993 hasta el 28 de julio de 1994 en una muestra de 183 pacientes que presentaron manifestaciones clínicas de lepra con o sin confirmación bacteriológica y que iniciaron por primera vez un tratamiento prescrito por un médico. La discapacidad se clasificó siguiendo los criterios de la OMS (15). Se consideraron lesiones precursoras de discapacidad las así establecidas a partir de estudios fisiopatológicos (32-35). En concreto, nos referimos a las infiltraciones en la cara o en la región perior- bitaria, las lesiones cutáneas alrededor del ojo, las señales de lesión avanzada en el trayecto del nervio (engrosamiento con dolor provocado o espontáneo, lesión cutánea extensa con anestesia, anestesia en una zona extensa, etc.) y los resultados de la infección secundaria. La exposición fue evaluada considerando la influencia de las redes sociales de apoyo y de los servicios de salud en la manera en que el paciente se enfrentó a la enfermedad.

Los pacientes seleccionados tenían de 20 a 70 años de edad, eran residentes de Recife y entre noviembre de 1993 y julio de 1994 consultaron por primera vez o estaban completando un proceso diagnóstico iniciado anteriormente en los servicios estatales de dermatología sanitaria del Centro Integrado de Salud Amaury de Medeiros o del Centro de Salud Agamenon Magalhaes, unidades de referencia de las regiones politicoadministrativas tercera, cuarta y sexta. Todos los días, los pacientes que cumplían los requisitos de edad y residencia se interrogaban para saber si estaban consultando por primera vez o completando un proceso diagnóstico iniciado anteriormente. Se excluyeron del estudio tres pacientes que estaban reintegrándose al programa después de abandonar el tratamiento, uno que fue dado de alta por curación, así como otro que fue transferido a otro servicio sanitario y 25 que no habían iniciado el tratamiento al término del período de muestreo. Por último, se consideraron "casos" 64 pacientes elegibles con discapacidad o lesiones precursoras de discapacidad. Los 119 pacientes restantes fueron considerados "controles".

Se elaboró un instrumento que se ajustó para recoger informaciones sobre a) las características demográficas como la edad, el sexo, los antecedentes de migración, la escolaridad, el empleo, la posición en la familia y el acceso a los medios de comunicación; b) la disponibilidad de apoyo social; c) el manejo habitual de los problemas de salud; y d) el acceso a los servicios de salud. Las opiniones personales respecto a manifestaciones corporales, modificaciones ocurridas en la vida del paciente e información sobre la enfermedad (sospecha de lepra, forma en que adquirió la enfermedad, proyección de consecuencias futuras, expectativas de curación) fueron recolectadas utilizando una guía semiestructurada y una grabadora magnetofónica y luego fueron descodificadas mediante la extracción de "núcleos de sentido" (36), dando origen así a una matriz de variables dicotómicas.

Los datos fueron recolectados en los servicios de salud y en los domicilios de los pacientes (con autorización previa) por medio de entrevistas semiestructuradas con la ayuda de un prontuario revisado. Las entrevistas estuvieron a cargo de una enfermera y una asistenta social que habían recibido capacitación en esas tareas. Para controlar el sesgo de clasificación (con respecto a la exposición), se explicó concienzudamente a las entrevistadoras la necesidad de dar el mismo tratamiento a todos los pacientes. Prácticamente todos los pacientes seleccionados accedieron a ser entrevistados. Solo tres rehusaron.

Para reconstruir la trayectoria que llevó al paciente al diagnóstico, usamos la técnica de las matrices introducida por la escuela de geografía del tiempo, que inició Haggerstrand con sus modelos espaciotemporales del comportamiento humano (37). En nuestros formularios sustituimos el concepto original de espacio físico por el de espacio social, conformado por las diversas alternativas de ayuda disponibles y observamos el movimiento de cada paciente en el eje temporal (figura 1). Así pudimos observar simultáneamente el movimiento del paciente a lo largo de distintos pasos alternativos y el tiempo lógico (no necesariamente cronológico) del proceso de búsqueda de ayuda (38). Esos perfiles fueron traducidos a variables discretas o continuas que se integraron en una base de datos.

Se efectuó un control sistemático de la calidad. Los registros de cada paciente fueron analizados cuidadosamente en busca de indicios para nuevas exploraciones y se revisaron su integridad, legibilidad y consistencia interna. 
FIGURA 1. Percepción y manejo de la enfermedad de Hansen por los enfermos antes del diagnóstico profesional. Recife, Brasil, 1994

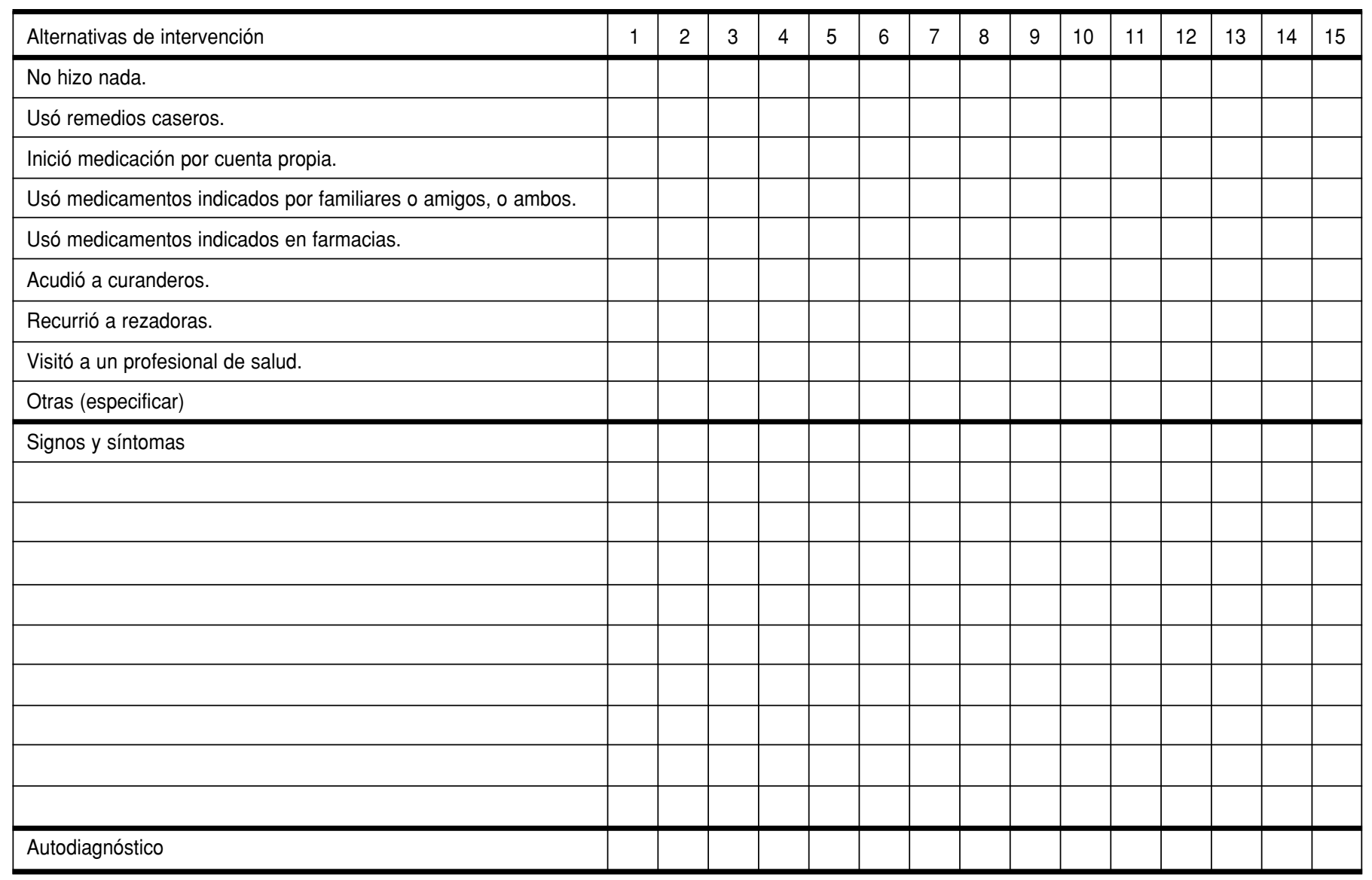

En el análisis se objetivó: a) identificar las variables capaces de discriminar entre grupos, aunque las que tenían mayor nexo funcional con el proceso investigado continuaron incorporadas al resto del análisis independientemente de si tenían o no significación estadística; b) estimar la probabilidad de pertenecer al grupo de casos mediante el cálculo de la razón de desigualdad; c) explorar el comportamiento de los dos grupos en relación con el tiempo transcurrido antes de la primera consulta y antes de iniciar el tratamiento; y d) identificar y controlar la influencia del sexo, la edad, la escolaridad, la experiencia previa con pacientes de lepra y la forma clínica de la enfermedad.

La significación estadística de la asociación (intervalo de confianza de 95\%, IC95\%) se determinó mediante la prueba de ji cuadrado $\left(\chi^{2}\right)$ o la prueba exacta de Fisher (cuando fue necesaria por haber pocos elementos en alguna de las casillas). El término tendencia se utilizó para describir resultados en los que se observaron diferencias que no tenían significación estadística. Al intentar comprender el significado de los datos, se usó el modelo interpretativo de las decisiones humanas propuesto por Allen (26).

Según la capacidad potencial de ejecución de actividades relacionadas con el control de la enfermedad de Hansen, los servicios sanitarios usados por los pacientes en el proceso que les llevó a obtener el diagnóstico fueron clasificados de la siguiente forma: nivel 1, consulta médica en clínicas básicas; nivel 2, consulta médica en clínicas básicas y en alguna especialidad; y nivel 3, consulta a centros de referencia para el tratamiento de la enfermedad.

Algunas variables de comportamiento limítrofe (que en general presentaban intervalos de confianza de mayor amplitud) mostraron que entre los dos grupos había diferencias que desaparecían o fuertes tendencias cuya significación se hacía evidente al ajustar respecto a una o más de las variables de control. Esto sugiere que a pesar de que el tamaño muestral permitió obtener datos importantes sobre el proceso estudiado, no fue suficiente para detectar diferencias de menor magnitud.

\section{RESULTADOS}

De los pacientes clasificados como casos, 19 (30\%) tenían discapacidad (de 
grado 2 en 13 pacientes) y $45(70 \%)$ tenían lesiones precursoras de discapacidad (en 25 pacientes correspondían a algún tipo de lesión avanzada de uno o más troncos nerviosos) (cuadro 1). En más de la mitad de los casos (36 de 64) se diagnosticó la forma clínica dimorfa. Entre los controles la forma clínica más frecuente fue la tuberculoide $(48 / 119=$ $40 \%$ ). Menos frecuentes fueron la forma clínica indeterminada $(24 / 119=$ $29 \%)$, la dimorfa $(32 / 119=27 \%)$ y la virchowiana $(5 / 119=4 \%)$.

Los casos y los controles fueron similares con respecto a la edad (mediana de los casos, 38 años, con un mínimo de 25 y un máximo de 47; mediana de los controles, 35 , con recorrido de 26 a 47 años). En cuanto a la forma habitual de actuar frente a la enfermedad, $80 \%$ en ambos grupos usaban remedios caseros y se automedicaban, lo que muestra una tendencia predominante a ensayar acciones alternativas antes de buscar atención profesional. La mayoría (más de $90 \%$ en cada grupo) contaba con redes de apoyo social formadas por familiares, amigos, vecinos, etc., que le ayudaban a enfrentar las situaciones propias de la vida cotidiana.

De los casos, $53 \%$ eran varones, $19 \%$ de ellos analfabetos. La mediana de escolaridad era de 4 años, con un recorrido de 1 a 6 años. Una mayor proporción de los casos (34\% comparado con $18 \%$ de los controles) había vivido la mayor parte de su vida en Recife; $89 \%$ miraban televisión y $87 \%$ escuchaban programas de radio. Entre los que contaban con una red social, $81 \%$ recibían apoyo financiero y otros materiales. Una cuarta parte de ellos consultaban a un médico solamente cuando pensaban que tenían una enfermedad grave. De los que acudían a servicios

CUADRO 1. Pacientes con enfermedad de Hansen; clasificación de discapacidades y de lesiones precursoras de discapacidad. Recife, Brasil, 1994

\begin{tabular}{|c|c|c|c|c|c|c|c|}
\hline \multirow[b]{3}{*}{ Tipo de lesión } & \multicolumn{6}{|c|}{ Forma clínica } & \multirow[b]{3}{*}{ Total } \\
\hline & \multicolumn{2}{|c|}{ Tuberculoide } & \multicolumn{2}{|c|}{ Dimorfa } & \multicolumn{2}{|c|}{ Virchowiana } & \\
\hline & No. & $\%$ & No. & $\%$ & No. & $\%$ & \\
\hline \multicolumn{8}{|l|}{ Discapacidad de grado: } \\
\hline 1 & 1 & 5,3 & 3 & 15,7 & 1 & 5,3 & 5 \\
\hline 2 & - & - & 7 & 36,6 & 2 & 10,6 & 9 \\
\hline 1 у 2 & 1 & 5,3 & 2 & 10,6 & - & - & 3 \\
\hline 1 y $N$ & - & - & - & - & 1 & 5,3 & 1 \\
\hline 1,2 y N & - & - & - & - & 1 & 5,3 & 1 \\
\hline Subtotal & 2 & 10,6 & 12 & 62,9 & 5 & 26,5 & 19 \\
\hline \multicolumn{8}{|l|}{ Lesión precursora: } \\
\hline $\begin{array}{l}\text { la región periorbitaria } \\
\text { Lesión cutánea alrededor } \\
\text { del ojo o en la región }\end{array}$ & - & - & 5 & 11,1 & 4 & 8,9 & 9 \\
\hline $\begin{array}{l}\text { maxilar } \\
\text { Engrosamiento del trayecto }\end{array}$ & - & - & 7 & 15,6 & - & - & 7 \\
\hline $\begin{array}{l}\text { del nervio, con dolor } \\
\text { Dolor en el trayecto del }\end{array}$ & 2 & 4,4 & - & - & - & - & 2 \\
\hline $\begin{array}{l}\text { nervio } \\
\text { Lesión cutánea extensa }\end{array}$ & 7 & 15,6 & 7 & 15,6 & 1 & 2,2 & 15 \\
\hline $\begin{array}{l}\text { con anestesia } \\
\text { Ulceración y perforación }\end{array}$ & 4 & 8,9 & 4 & 8,9 & - & - & 8 \\
\hline $\begin{array}{l}\text { del tabique nasal } \\
\text { Parestesia o anestesia en }\end{array}$ & - & - & - & - & 1 & 2,2 & 1 \\
\hline $\begin{array}{l}\text { la región hipoténar } \\
\text { Hipoestesia de }\end{array}$ & 1 & 2,2 & 1 & 2,2 & - & - & 2 \\
\hline seguimientos corporales & - & - & - & - & 1 & 2,2 & 1 \\
\hline Subtotal & 14 & 31,1 & 24 & 53,4 & 7 & 15,5 & 45 \\
\hline Total & 16 & - & 36 & - & 12 & - & 64 \\
\hline
\end{tabular}

de salud, $82 \%$ escogieron unidades de la red de salud pública y filantrópica; $57 \%$ de estos dijeron que su elección se basaba en la accesibilidad geográfica.

De los controles, $68 \%$ eran mujeres, $7 \%$ de ellas analfabetas. La mediana de escolaridad en ellas fue de 5 años, con un recorrido de 3 a 8 años; $97 \%$ veían televisión y $93 \%$ escuchaban la radio. Entre los controles que contaban con una red social, $61 \%$ recibían apoyo solidario y consejos. Cerca de $68 \%$ consultaron a un médico después del fracaso de la terapia utilizada por cuenta propia o de seguir los consejos de amigos, independientemente del carácter progresivo de la enfermedad. De los enfermos que utilizaron los servicios de salud, $26 \%$ acudieron a los servicios privados de la seguridad social.

Los dos subgrupos que constituyeron los casos presentaron características semejantes con excepción de que una proporción significativamente mayor de los que tenían lesiones precursoras de discapacidad siempre vivieron en Recife $(P=0,011)$ y se automedicaban cuando se sentían enfermos $(P=0,005)$.

Los casos y controles expresaron opiniones bastante concordantes sobre la lepra. Si se examinan ambos grupos en conjunto, $69 \%$ de los pacientes tenían una expectativa de que la enfermedad se pudiera curar; $24 \%$ pensaban que el aislamiento, la parálisis, la deformidad y la mutilación eran posibles consecuencias de la lepra, y $27 \%$ veían en los servicios de salud una importante fuente de información sobre esa enfermedad (27\%). Solamente $15 \%$ de los pacientes pudieron dar alguna explicación de cómo se contrae la lepra, entre ellos una proporción levemente más elevada de los controles (19\%) en comparación con los casos ( $9 \%$ ). Además, $62 \%$ no habían tenido ninguna experiencia previa con enfermos de lepra.

En los casos, que estaban más afectados por las lesiones y otros síntomas cuando descubrieron que tenían la enfermedad, fue marcado el carácter progresivo de las lesiones. En una proporción significativamente mayor de los controles $(P=0,002)$, la primera manifestación notada fue una lesión cutá- 
nea única con pérdida de la sensibilidad local (cuadro 2).

Como muestra el cuadro 3, en el momento de descubrir la enfermedad tanto los casos como los controles se comportaron de forma similar en lo que se refiere a expresar alguna sospecha sobre el diagnóstico. Sin embargo, antes de la primera consulta médica se estableció una diferencia acentuada entre los dos grupos, pues los casos presentaron una mayor frecuencia de cambios relacionados con esa sospecha. Muy pocos pacientes pensaban que tenían la enfermedad de Hansen, pero durante el período anterior a la

CUADRO 2. Características de las manifestaciones clínicas en pacientes con enfermedad de Hansen con y sin discapacidades o lesiones precursoras de discapacidades. Recife, Brasil, 1994

\begin{tabular}{lrrrrr}
\hline Manifestación clínica percibida & Casos & Controles & OR & IC & $P$ \\
\hline Lesión cutánea única & $3 / 64$ & $84 / 119$ & 0,23 & $0,12-0,47$ & 0,000 \\
$\begin{array}{l}\text { Múltiples lesiones cutáneas } \\
\begin{array}{l}\text { Alteración de la sensibilidad } \\
\text { en la lesión percibida }\end{array}\end{array}$ & $10 / 64$ & $7 / 119$ & 2,96 & $0,96-9,30$ & 0,031 \\
$\begin{array}{l}\text { Progresión de las manifestaciones } \\
\text { antes de la primera consulta }\end{array}$ & $5 / 64$ & $28 / 119$ & 0,28 & $0,09-0,81$ & 0,008 \\
$\begin{array}{l}\text { Progresión de las manifestaciones } \\
\text { entre la primera consulta y el }\end{array}$ & $64 / 64$ & $103 / 119$ & - & - & 0,002 \\
inicio del tratamiento & & & & & \\
\hline
\end{tabular}

$\mathrm{OR}=$ odds ratio o razón de posibilidades.

IC = intervalo de confianza.

CUADRO 3. Características del autodiagnóstico en pacientes con enfermedad de Hansen con y sin discapacidades o lesiones precursoras de discapacidades. Recife, Brasil, 1994

\begin{tabular}{|c|c|c|c|c|c|}
\hline Autodiagnóstico & Casos & Controles & OR & IC & $P$ \\
\hline $\begin{array}{l}\text { Sospechó que tenía alguna } \\
\text { enfermedad desde los primeros } \\
\text { síntomas }\end{array}$ & $43 / 64$ & $75 / 119$ & 1,20 & $0,60-2,40$ & 0,576 \\
\hline $\begin{array}{l}\text { Cambió de opinión antes de la } \\
\text { primera consulta }\end{array}$ & $39 / 64$ & $50 / 119$ & 2,15 & $1,10-4,23$ & 0,015 \\
\hline $\begin{array}{l}\text { Cambió de opinión entre la } \\
\text { primera consulta y el inicio del } \\
\text { tratamiento }\end{array}$ & $20 / 63^{a}$ & $37 / 116^{a}$ & 0,99 & $0,48-2,03$ & 0,983 \\
\hline $\begin{array}{l}\text { Sospechó enfermedad de Hansen: } \\
\text { al percibir que estaba enfermo } \\
\text { entre la primera percepción de }\end{array}$ & $1 / 64$ & $4 / 119$ & 0,46 & $0,01-4,76^{b}$ & $0,427^{c}$ \\
\hline $\begin{array}{l}\text { enfermedad y la primera consulta } \\
\text { entre la primera consulta y el inicio } \\
\text { del tratamiento }\end{array}$ & $\begin{array}{l}7 / 64 \\
51 / 64\end{array}$ & $30 / 119$ & 0,36 & $0,14-0,94$ & 0,022 \\
\hline $\begin{array}{l}\text { Tenía antecedentes de enfermedad } \\
\text { de Hansen que motivaron su sospecha }\end{array}$ & $7 / 64$ & $17 / 119$ & 0,19 & $0,03-0,93$ & 0,018 \\
\hline $\begin{array}{l}\text { La información que le dio un } \\
\text { profesional sobre la enfermedad de } \\
\text { Hansen motivó su sospecha }\end{array}$ & $41 / 64$ & $60 / 119$ & 1,75 & $0,89-3,46$ & 0,077 \\
\hline
\end{tabular}

$\mathrm{OR}=$ odds ratio o razón de posibilidades.

$\mathrm{IC}=$ intervalo de confianza.

a Excluidos los casos sin información.

b Límites exactos.

c Prueba exacta de Fisher.

primera consulta, ya una proporción significativamente mayor de los controles admitía esa posibilidad $(29 \%$ frente a $13 \%$ de los casos).

En los dos grupos, la mayor frecuencia de autodiagnóstico de lepra ocurrió después de consultar con el servicio de dermatología sanitaria, especialmente en los casos, por la información obtenida del profesional que los atendió. Un grupo de cerca de $10 \%$ de los pacientes, de los cuales $90 \%$ eran controles, sospecharon que tenían la enfermedad de Hansen debido a antecedentes de esa dolencia. Por lo general, esta sospecha precedió a su primera visita al servicio de salud. Sin embargo, $11 \%$ de los casos y $6 \%$ de los controles nunca aceptaron el diagnóstico de enfermedad de Hansen hasta el momento de empezar el tratamiento.

En lo referente a las percepciones de los pacientes estudiados sobre su dolencia, el cuadro 4 muestra que cerca de $56 \%$ de ellos (63\% de los casos y $52 \%$ de los controles) no tenían idea alguna de cómo contrajeron la enfermedad. Del pequeño número que opinó correctamente sobre la forma en que contrajeron la enfermedad, la mayor parte eran controles. No se encontraron diferencias entre los dos grupos en cuanto a sus expectativas de curarse $(69 \%)$ y a su pronóstico del desarrollo de discapacidades estigmatizantes $(24 \%)$. Pertenecer al grupo de casos se asoció con sentirse enfermo en algún momento del estudio y con considerar que la enfermedad tenía implicaciones negativas para su vida, principalmente como factor que limitaba sus actividades. Entre los controles predominó la idea de que la enfermedad modificaría sus relaciones personales.

Como indica el cuadro 5, entre los casos se observó una tendencia a automedicarse y a usar medicamentos recomendados y remedios caseros, lo cual redundó en una proporción mayor de intervenciones que en los controles, independientemente de la orientación profesional recibida. La mayoría de las veces, la primera consulta de los casos en el servicio de salud fue motivada por la progresión de sus síntomas. Entre los controles, la 
CUADRO 4. Percepciones sobre su dolencia en pacientes con enfermedad de Hansen con y sin discapacidades o lesiones precursoras de discapacidades. Recife, Brasil, 1994

\begin{tabular}{lccccc}
\hline \multicolumn{1}{c}{ Percepción } & Casos & Controles & OR & IC & $P$ \\
\hline Opinión correcta sobre la forma & & & & & \\
$\quad$ en que se enfermó & $4 / 64$ & $28 / 119$ & 0,22 & $0,06-0,70$ & 0,003 \\
Expectativa de cura & $40 / 59^{\mathrm{a}}$ & $89 / 115^{\mathrm{a}}$ & 0,62 & $0,29-1,32$ & 0,172 \\
Preveía consecuencias estigmatizantes & $17 / 64$ & $26 / 119$ & 1,29 & $0,60-2,79$ & 0,474 \\
Se sentía enfermo & $30 / 64$ & $21 / 119$ & 4,12 & $1,96-8,68$ & 0,000 \\
La enfermedad cambió su vida & $34 / 64$ & $41 / 119$ & 2,16 & $1,10-4,23$ & 0,014 \\
La enfermedad limitó su desempeño & $16 / 34$ & $4 / 41$ & 8,22 & $2,16-37,62^{\mathrm{b}}$ & 0,000 \\
La enfermedad afectó sus relaciones & & & & & \\
personales & $4 / 34$ & $15 / 41$ & 0,23 & $0,06-0,89$ & 0,014 \\
\hline
\end{tabular}

$\mathrm{OR}=$ odds ratio 0 razón de posibilidades

$I C=$ intervalo de confianza.

a Excluidos los casos sin información

${ }^{b}$ Límites exactos.

CUADRO 5. Características del manejo de la enfermedad de Hansen por pacientes con y sin discapacidades o lesiones precursoras de discapacidad. Recife, Brasil, 1994

\begin{tabular}{lccccc}
\hline \multicolumn{1}{c}{ Manejo } & Casos & Controles & OR & IC & $P$ \\
\hline $\begin{array}{c}\text { Tomó medidas sin la opinión de un profesional: } \\
\quad \text { A la primera percepción de enfermedad }\end{array}$ & $13 / 64$ & $15 / 119$ & 1,77 & $0,73-4,29$ & 0,168 \\
$\begin{array}{c}\text { Entre la primera percepción y la } \\
\text { primera consulta }\end{array}$ & $37 / 64$ & $46 / 119$ & 2,17 & $1,11-4,26$ & 0,013 \\
$\begin{array}{c}\text { Entre la primera consulta y el inicio del } \\
\text { tratamiento }\end{array}$ & $14 / 64$ & $12 / 116$ & 2,43 & $0,96-6,15$ & 0,035 \\
$\begin{array}{c}\text { Acudió a la primera consulta debido a otra } \\
\text { dolencia }\end{array}$ & $3 / 64$ & $17 / 119$ & 0,30 & $0,07-1,14$ & 0,048 \\
$\begin{array}{c}\text { Acudió a la primera consulta porque las } \\
\text { manifestaciones progresaron }\end{array}$ & $45 / 61$ & $46 / 102$ & 3,42 & $1,62-7,32$ & 0,000 \\
$\begin{array}{c}\text { Pasó un máximo de 9 meses entre la } \\
\text { primera percepción de enfermedad y }\end{array}$ & & & & & \\
$\quad \begin{array}{l}\text { la primera consulta } \\
\text { Acudió a Dermatología Sanitaria porque }\end{array}$ & $31 / 57^{\mathrm{a}}$ & $60 / 109 \mathrm{a}$ & 0,97 & $0,48-1,96$ & 0,935 \\
$\begin{array}{c}\text { sospechaba enfermedad de Hansen } \\
\text { Pasó un máximo de 12 meses entre la } \\
\text { primera percepción de enfermedad y } \\
\quad \text { su consulta en Dermatología Sanitaria }\end{array}$ & $12 / 64$ & $46 / 119$ & 0,37 & $0,16-0,81$ & 0,006 \\
\hline
\end{tabular}

$\mathrm{OR}=$ odds ratio 0 razón de posibilidades.

$\mathrm{IC}=$ intervalo de confianza.

${ }^{a}$ Excluidos los casos sin información.

sospecha de que tenían la enfermedad de Hansen fue un factor importante que los impulsó a acudir al servicio de dermatología sanitaria.

En lo que se refiere al tiempo que transcurrió entre la percepción inicial de la enfermedad y la primera consulta, la mediana fue de 9 meses, con un recorrido de 4 a 23 meses en los casos y de 3,5 a 23 meses en los controles $(P=0,765)$. Comparando el comportamiento de casos y controles según la forma clínica de lepra diagnosticada, se notó que los casos infectados con la forma virchowiana dejaron transcurrir un intervalo más largo que los controles antes de la primera consulta.

Ambos grupos tuvieron un comportamiento semejante en relación con el tiempo transcurrido entre la percepción inicial de la enfermedad y la primera consulta en el servicio de dermatología sanitaria. La mediana fue de
12 meses en los casos (7 a 24 meses) y de 16 meses en los controles (6 a 34 meses), $P=0,717$. La similitud se mantuvo cuando se evaluó el comportamiento de los dos grupos, según la forma clínica de la enfermedad.

La asociación entre variables es más fuerte cuando no se tiene en cuenta la forma clínica de la enfermedad. Si se excluye la forma indeterminada, que solo se diagnosticó en los controles, la forma de lepra produce modificaciones de pequeña magnitud, que no afectan a la significación de las relaciones observadas, en la percepción de los signos y síntomas de la dolencia, sospecha de tener la enfermedad y manera de actuar al respecto.

En relación con los servicios de salud, 54\% de los pacientes tuvieron su primera consulta en un servicio de nivel 2. El cuadro 6 muestra que no hubo diferencias entre casos y controles en cuanto a la utilización de un servicio de nivel 2 en la primera consulta. Una proporción significativamente mayor de los controles acudió a las unidades de salud de uso habitual.

Los controles mostraron una tendencia leve a establecer un mayor número de contactos con los servicios de salud antes de iniciar el tratamiento, con una mediana de 10 contactos (recorrido de 7 a 13), mientras que los casos tuvieron una mediana de nueve contactos (6 a 12), $P=0,141$. Cerca de $26 \%$ de los pacientes tuvieron acceso sin mayores dificultades a la consulta médica y a los medios diagnósticos complementarios. Casi no hubo diferencias entre los dos grupos, especialmente si se considera que no hubo limitaciones en su acceso a los servicios de salud. Del 74\% que encontró obstáculos al acudir a esos servicios, los casos fueron un poco menos afectados por ellos.

La eficiencia de la detección de la enfermedad de Hansen en la primera consulta se evaluó según el nivel de complejidad del servicio consultado. Se tuvieron como criterios la emisión de una hipótesis diagnóstica por parte del profesional y las acciones sugeridas por él a los pacientes, a quienes no se les mencionó una hipótesis diagnóstica de lepra pero sí la posibilidad de 
CUADRO 6. Interacción con los servicios de salud de pacientes con enfermedad de Hansen con y sin discapacidades o lesiones precursoras de discapacidad. Recife, Brasil, 1994

\begin{tabular}{llllll}
\hline \multicolumn{1}{c}{ Servicio de salud } & Casos & Controles & OR & IC & $P$ \\
\hline $\begin{array}{l}\text { Primera consulta en un servicio de nivel } 2 \\
\text { Primera consulta en el servicio habitual- }\end{array}$ & $31 / 64$ & $68 / 118^{\mathrm{a}}$ & 0,69 & $0,36-1,34$ & 0,236 \\
$\quad$ mente utilizado & $26 / 64$ & $68 / 118^{\mathrm{a}}$ & 0,50 & $0,26-0,98$ & 0,029 \\
$\begin{array}{l}\text { Número máximo de 10 contactos antes de } \\
\text { iniciar el tratamiento }\end{array}$ & $35 / 64$ & $82 / 119$ & 0,54 & $0,28-1,08$ & 0,057 \\
$\begin{array}{l}\text { No sintieron limitaciones de acceso a los } \\
\text { servicios }\end{array}$ & $20 / 64$ & $27 / 119$ & 1,55 & $0,74-3,23$ & 0,207 \\
$\begin{array}{l}\text { Sintieron limitaciones de acceso en hasta } \\
12 \% \text { de los contactos }\end{array}$ & $13 / 44$ & $17 / 92$ & 1,85 & $0,73-4,65$ & 0,147 \\
\hline
\end{tabular}

$\mathrm{OR}=$ odds ratio 0 razón de posibilidades.

IC = intervalo de confianza.

a Excluidos los casos sin información.

ese diagnóstico, remitiéndolos al Servicio de Dermatología Sanitaria y solicitando biopsias y baciloscopias. Si bien estos criterios tenían limitaciones, permitieron una aproximación preliminar a la evaluación de la eficiencia diagnóstica (figura 2).

La eficiencia de los servicios por lo general fue pobre, pero los menos eficientes de todos fueron los de nivel 3, excepto para aquellos pacientes que tuvieron su primera consulta en el servicio de dermatología sanitaria. Los servicios de nivel 1 y nivel 2 fueron de calidad general similar, pero el dictamen de un diagnóstico tentativo de lepra fue bastante más importante en los de nivel 2.

En el curso de acción que llevó a los pacientes a conocer su diagnóstico, no se observaron diferencias importantes debidas al apoyo recibido de sus redes de relaciones sociales. Considerando ambos grupos en conjunto, $92 \%$ de los pacientes contaron con una red de apoyo durante su enfermedad, $51 \%$ recurrieron a la red habitualmente movilizada de familiares, amigos y vecinos y $68 \%$ recibieron ayuda de algún miembro de la red intradomiciliaria. En 30\%, los miembros de la red de apoyo sospechaban que la enfermedad era lepra. Entre los controles se observó una tendencia moderada a pedir ayuda a miembros de sus redes sociales, pero la participación de estas en el proceso del diagnóstico fue fundamental en una mayor proporción de los casos que de los controles. En con- junto, $94 \%$ indicaron que se les había aconsejado que buscaran atención profesional y $26 \%$, que se les habían sugerido diversos remedios caseros. fesional. Recife, Brasil, 1994
Nivel 1

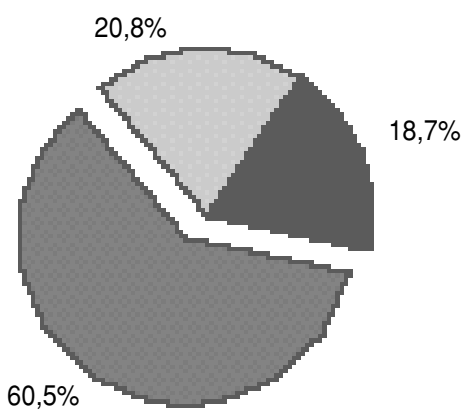

$\square$ Conducta que sugería la hipótesis

No se emitió diagnóstico

\section{DISCUSIÓN}

Solamente $18 \%$ de los pacientes dieron una explicación coherente de cómo enfermaron. Los casos, en particular, mostraron un pequeño número de modelos explicativos relacionados con la enfermedad, aunque habían cambiado más frecuentemente su autodiagnóstico e informado de experiencias previas con síntomas similares. Los profesionales de salud desempeñaron un papel importante en el autodiagnóstico de enfermedad de Hansen, si bien la formulación y comunicación de la hipótesis diagnóstica se efectuaron después de una larga trayectoria de contactos con los servicios de salud.

Los casos se sintieron más afectados que los controles por lesiones $\mathrm{u}$ otras manifestaciones físicas de la enfermedad. También fue más frecuente en los

FIGURA 2. Eficiencia del diagnóstico de enfermedad de Hansen en la primera consulta pro-

Nivel 2

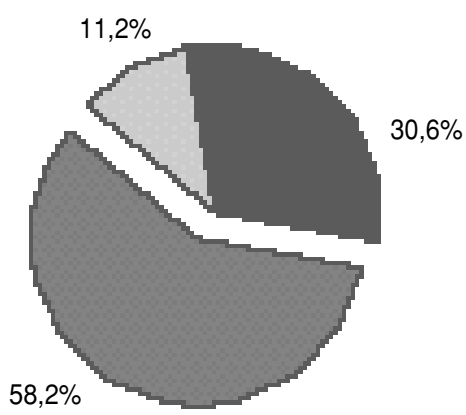

Nivel 3

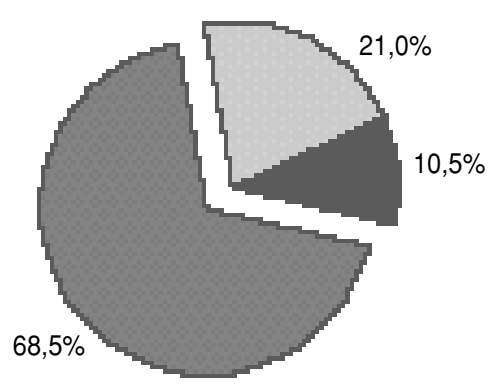

La hipótesis diagnóstica de enfermedad de Hansen se manifestó mediante:

Comunicación de la hipótesis diagnóstica al paciente 
casos quejarse de malestar y considerar que la enfermedad tendría repercusiones negativas en su vida. Por lo tanto, a pesar de que el tiempo cronológico transcurrido antes de la primera consulta con un profesional de la salud fue similar en casos y controles, los pasos que llevaron a los casos a comprender y manejar su situación sugieren la existencia de diferencias importantes en el "tiempo lógico", es decir que ha ocurrido un retraso relativo, ya que las quejas presentadas más bien señalaban la necesidad de buscar rápidamente atención profesional.

Además, si bien los casos solicitaron ayuda con menor frecuencia, la influencia de la red social fue fundamental para motivar a una mayor proporción de ellos a seguir buscando el diagnóstico definitivo, lo que sugiere menos espontaneidad en el proceso de tomar una decisión. Todo parece indicar que esos pacientes eran menos conscientes del riesgo a que estaban expuestos y que aun los antecedentes de la enfermedad no fueron razón suficiente para hacerles pensar que podían tener lepra y acudir con mayor celeridad a los servicios de salud. Otra forma de explicar su comportamiento es que, a pesar de considerar la posibilidad de tener enfermedad de Hansen, callaran por temor al rechazo social. Nuestro estudio sistemático no permitió confirmar esta posibilidad.

Los pacientes de ambos grupos expresaron opiniones positivas en cuanto a la probabilidad de curarse. ¿Había llegado el concepto de la incurabilidad verdaderamente a tener menor peso en las concepciones de estigmatización de la enfermedad? En
Santander, Colombia, se observó un comportamiento similar en un contexto sociocultural y epidemiológico diferente (12).

El proceso que podría llamarse "globalización" es un fenómeno social que se localiza, arraigándose en las prácticas cotidianas y redefiniéndolas en su especificidad. En este sentido, los medios de comunicación tienen una dimensión que trasciende su ámbito y hace posible que los valores, prácticas y reglas sociales sean constantemente evaluados y modificados a la luz de nueva información $(39,40)$. Los pacientes tenían acceso a los mensajes que se transmiten sistemáticamente en los medios de comunicación sobre descubrimientos científicos y soluciones tecnológicas para distintos problemas de salud; en consecuencia, a ellos podrían deberse sus expectativas de curación.

Por otra parte, al confrontar las necesidades sentidas por los pacientes de lepra con la forma como esta dolencia se trata en las instituciones de salud pública, se encontraron obstáculos importantes en la dificultad de acceso a la consulta médica y la baja prioridad que se otorga al problema en la red de atención. Estas deficiencias elevan el costo social y financiero de la enfermedad y quizá estimulan la búsqueda de soluciones alternativas.

La dinámica de la atención prestada a estos pacientes sugiere que, en Recife, los servicios de salud no están preparados para ejecutar las acciones que les competen para eliminar la lepra. Según el nivel respectivo de complejidad, esas acciones serían: para las unidades de los niveles 1 y 2, identificar los casos sospechados de lepra, notificarlos y remitir los pacientes a los servicios de dermatología sanitaria para confirmación y tratamiento. La falta de un sistema de referencia efectivo entre las unidades de salud amplía el número de contactos institucionales y retrasa el inicio del tratamiento, incluso para aquellos que ya han tenido un diagnóstico preliminar de enfermedad de Hansen.

En una zona donde la lepra es endémica y en expansión, es preocupante haber comprobado que existen dos tipos de invisibilidad de la enfermedad: 1) entre los pacientes, la baja frecuencia de modelos explicativos, espontáneamente formulados, aun en pacientes con antecedentes de la dolencia; y 2) lo que es aun más grave, las limitaciones encontradas en cuanto a detección entre los profesionales de la salud.

Los resultados de este estudio indican que es necesario introducir profundas modificaciones en el comportamiento de los diferentes actores involucrados, ya que este interfiere con las decisiones sobre el manejo individual o colectivo de la enfermedad. Ello constituye en sí mismo un factor de riesgo que puede contribuir a agravar las consecuencias físicas $y$ sociales de la lepra, la persistencia de su transmisión $y$, sin duda, a obstaculizar la meta de eliminarla como problema de salud pública.

Agradecimiento. Los autores expresan su reconocimiento a la asistenta social Suzette Maria Feitosa Brito y a la enfermera Cácia Mendes, responsables del trabajo sobre el terreno.

\section{REFERENCIAS}

1. Cvjetanovic B. The dynamics of bacterial infection. En: Anderson RM. The population dynamics of infectious diseases: theory and applications. London: Chapman and Hall; 1982: 38-66.

2. Albuquerque M de FPM, Barros HM, Ximenes RAA. A expansão da hanseníase no Nordeste brasileiro. Rev Saude Publica 1989;23: 101-116.
3. Brasil, Secretaria de Saúde de Pernambuco. Relatório anual das ações de controle da hanseníase em 1992. (Documento fotocopiado).

4. Minayo MC de S. Abordagem antropológica para avaliação de políticas sociais. Rev Saude Publica 1991;25:233-238.

5. Infante-Castañeda C. Bases para el estudio de la interacción familia-redes sociales-uso de servicios de salud. Salud Publica Mex 1988;30: 175-196.

6. Cohn A, Nunes E, Jacobi PR, Karsch US. O accesso em discussão: o viés da racionalidade e o viés da carência. En: Cohn A. A saúde como direito e como serviço. São Paulo: Cortez Editora; 1991:67-94.

7. Lindbladh E, Lyttkkens $\mathrm{CH}$, Hanson BS, Östergre P, Isacsson S, Lindgren B. An eco- 
nomic and sociological interpretation of social differences in health-related behaviour: an encounter as a guide to social epidemiology. Soc Sci Med 1996;43:1817-1827.

8. Berlinguer G. A doença como perigo. En: $A$ doença. São Paulo: CEBES-Hucitec; 1988: 73-93.

9. Brasil, Ministério de Saúde, Secretaria Especial de Programas Especiais de Saúde, Divisão Nacional de Dermatologia Sanitária (DNDS). Controle da hanseníase: uma proposta de integração ensino-serviço. Rio de Janeiro: DNDS/ NUTES; 1989.

10. Sevilla E. Entre expertos y dolientes. En: Los mutilados del oprobio. Santa Fe de Bogotá: Tercer Mundo Editoriales/Colcultura; 1995:21-36.

11. Feliciano K, Sevilla E, Alzate A. A study of the behavioral factors related to the occurrence of disabilities among leprosy patients of an endemic province of Colombia: Final report of Research Project TDR/WHO-ID:900687. Universidad del Valle, Cali, Colombia; 1992.

12. Srinivasan H. Newer tasks for leprosy workers. Indian J Lepr 1990;62:1-5.

13. Ridley DS, Jopling WH. Classification of leprosy according to immunity, a five-group system. Int J Lepr 1966;34(3):332-359.

14. Fine PEM. Immunogenetics of susceptibility to leprosy, tuberculosis, and leishmaniasis, an epidemiological perspective. Int J Lepr 1981; 49:437-454.

15. World Health Organization. A guide to leprosy control. 2a ed. Geneva: WHO; 1995.

16. Meyers WM. Leprosy. Dermatol Clin 1992; 10:73-96.

17. Vries RRP. Genetic control of immunopathology induced by Mycobacterium leprae. Am J Trop Med Hyg 1991;44:12-16.

18. Kaufmann A, Mariam SSG, Neville J. The social dimension of leprosy. Reimpresión. London: International Federation of Anti-Leprosy Associations (ILEP); 1986

19. Rosenstock IM. Por qué la gente utiliza los servicios de salud. En: White KL, Frenk J,
Ordóñez C, Paganini JM, Starfield B, eds. Investigaciones sobre servicios de salud: una antología. Washington, DC: Organización Panamericana de la Salud; 1992:405-424. (Publicación científica 534).

20. Sevilla E, Olaya J, Feliciano K. Dueños de sí y de sus deseos: la conducta sexual de los colombianos y su vulnerabilidad al VIH. Cali, Colombia: Universidad del Valle; 1994. (Informe final elaborado para el Instituto de Seguridad Social de Colombia).

21. Vellozo VRO, Souza RG. Acesso e hierarquização: um caminho (re)construído. En: Bodstein R. Serviços locais de saúde: construção de atores e politicas. Rio de Janeiro: RelumeDumará;1993:97-116.

22. Ferreira J. O corpo sígnico. En: Alves PC, Minayo MCS, eds. Saúde e doença: um olhar antropológico. Rio de Janeiro: Editora FIOCRUZ; 1994:101-112.

23. Rosenstock IM. Prevention of illness and maintenance of health. En: Kosa J, Zola IK. Poverty and health: a sociological analysis. Rev ed. Cambridge: Harvard University Press; 1975:193-223.

24. Adami NP. Acesso, utilização e aceitação dos serviços de saúde de dermatologia de um centro de saúde escola sob o modo de ver dos hansenianos. Rev Latinoam Enferm 1993;11-19.

25. Kroeger A. Anthropological and sociomedical health care research in developing countries. Soc Sci Med 1983;17:147-161.

26. Allen PM. Towards a new science of the complex system. En: The science and praxis of complexity: contributions of the symposium held at Montpellier, France, 1-9 May, 1984. Tokyo: The United Nations University; 1985.

27. Laplantine F. Questões de método. En: Antropologia da doença. São Paulo: Martins Fontes Editora; 1994:11-48.

28. Valla VV. A construção desigual do conhecimento e o controle social dos serviços de saúde . En: Valla VV, Stotz EN, eds. Participação popular, educação e saúde: teoria e prá- tica. Rio de Janeiro: Relume-Dumará; 1993 87-100.

29. Habermas J. Notas programáticas para a fundamentação de uma ética do discurso. En: Consciência moral e agir comunicativo. Rio de Janeiro: Tempo Brasileiro 1989:61-142.

30. Rodrigues JC. Saúde e significado. En: Antropologia do poder. Rio de Janeiro: Terra Nova Editora; 1991:103-118.

31. Bachelard G. A distensão e o nada. En: A dialética da duração. São Paulo: Ática; 1988:11-35.

32. Bryceson A, Pfaltzgraff RE. Complications due to nerve damage. En: Leprosy. 2a ed. New York: Churchill Livingstone; 1980:77-88.

33. Brand PW, Fritschi EP. Rehabilitation in leprosy. En: Hastings RC, ed. Leprosy. New York: Churchill Livingstone; 1985:287-319.

34. Job CK. Nerve damage in Hansen's disease, Part I. The Star (Carville, LA) 1990;50(2):11-15; Part II, The Star 1990;50(3):5-6.

35. Krotoski JB. Peripheral neuropathy and examination of the hands. The Star 1991;50 (5):1-5.

36. Minayo MC de S. Fase de análise ou tratamento do material. En: O desafio do conhecimento: pesquisas qualitativas em saúde. Rio de Janeiro: Hucitec-Abrasco;1992:197-248.

37. Parkes D, Thrift N. Time-geography: the Lund approach. En: Times, spaces, and places, a chronogeographic perspective. New York: John Wiley \& Sons; 1980:243-278.

38. Fabrega H. Towards a model of illness behavior. Med Care 1973;11:470-484.

39. Giddens A. The consequences of modernity. Stanford, California: Stanford University Press; 1990.

40. Ortiz R. Cultura e sociedade global. En: Mundialização e cultura. São Paulo: Editora Brasiliense; 1994:13-34.

Manuscrito recibido el 21 de julio de 1995 y aceptado para publicación en versión revisada el 4 de marzo de 1997.
ABSTRACT

\section{Perceptions regarding leprosy and resulting handicaps prior to diagnosis in Recife, Brazil}

This article reports on a case-control study conducted in Recife, Brazil, between November 1993 and July 1994, to determine how leprosy patients' perceptions and notions influence disease management and use of health services. The sample was composed of 183 residents of Recife between the ages of 20 and 70 years who sought diagnostic services in the dermatology clinics of two referral centers situated in the third, fourth, and sixth political and administrative regions. Sixty-four patients having handicaps or their precursor lesions were classified as cases; the remaining 119 were used as controls. All were diagnosed during the study period. For the analysis, adjustments were made for sex, age, schooling, and a previous history of Hansen's disease among patients.

The study revealed the simultaneous presence of two types of "invisibility" of the disease in an area where endemicity is increasing: 1) for patients in both groups, the low frequency of spontaneous explanatory models related to the illness, even in the presence of disease, and 2) for health professionals, the limitations of detection methods. Since such deficiencies affect decisions bearing on individual and collective disease management, they are a risk factor in and of themselves and stand in the way of eliminating leprosy as a public health problem. 